\section{Phenotypic variation in type II isolated growth hormone deficiency}

Type II isolated growth hormone deficiency (IGHD-II) is an autosomal-dominant inherited disorder with a variable phenotype. A new European study enrolled individuals with IGHD-II caused by two different mis-sense mutations that result in amino-acid substitutions in growth hormone (GH). Salemi et al. report the clinical and cellular effects of $\mathrm{P} 89 \mathrm{~L}$ mutation, compared with those of the most common mis-sense mutation $\mathrm{R} 183 \mathrm{H}$, and show that the P89L mutation compromises secretion of other proteins in addition to $\mathrm{GH}$, perhaps accounting for the severe phenotypes seen in affected individuals.

P89L mutations were identified in 12 IGHD-II individuals (from four families), and their clinical outcomes compared with those of 17 IGHD-II individuals (from five families) with $\mathrm{R} 183 \mathrm{H}$ mutations. Children with the P89L mutation showed growth deficits earlier than those with $\mathrm{R} 183 \mathrm{H}$ and tended to develop other pituitary hormone deficiencies; eight eventually developed deficiencies in TSH or adrenocorticotropin secretion, associated with pituitary hypoplasia.

$\mathrm{R} 183 \mathrm{H}$ mutation reduced intracellular accumulation and secretion of GH by about $50 \%$ in vitro compared with wild-type, but did not affect adrenocorticotropin secretion. By contrast, almost no secretion of $\mathrm{GH}$ was seen with P89L mutation, despite intracellular accumulation of P89L GH in the secretory apparatus that eventually reached similar levels to wild-type. Adrenocorticotropin secretion was also drastically reduced, implying a profound, general effect on cellular secretory pathways; the authors speculate that protein misfolding in the endoplasmic reticulum obstructs traffic of other proteins as well.

Caroline Barranco

Original article Salemi S et al. (2005) Variability of isolated autosomal dominant GH deficiency (IGHD II): impact of the P89L GH mutation on clinical follow-up and GH secretion. Eur J Endocrinol 153: 791-802

\section{Androgen deprivation therapy and BMD loss}

Several studies have suggested that androgen deprivation therapy (ADT), often used in the treatment of prostate cancer, is associated with loss of BMD. Greenspan et al. have conducted a 12-month, prospective, US study comparing the rate of bone loss in men with prostate cancer versus healthy men, and in men initiating ADT versus men who had received chronic ADT (for $\geq 6$ months).

The nonrandomized study enrolled 152 men (mean age 68 years) with nonmetastatic prostate cancer and 43 healthy, age-matched controls. The men with cancer were categorized as having no ADT $(n=72)$, acute ADT (mean duration $2.9 \pm 1.5$ months, $n=30$ ) or chronic ADT (mean duration 33.2 \pm 33.4 months, $n=50$ ). BMD of the hip, wrist, total body, and spine was measured at baseline and at 6 and 12 months, by dual-energy $\mathrm{X}$-ray absorptiometry.

At 12 months, men in the acute ADT group showed a statistically significant reduction in BMD of the total hip, trochanter, wrist, spine and total body (all $P<0.05$ ), compared with baseline BMDs. Men in the age-matched control and chronic ADT group showed a reduction in BMD only of the wrist $(P<0.05)$, and men in the no-ADT group showed no reductions in BMD.

Bone loss was greatest in the first year of ADT, indicating that men initiating treatment are at the highest risk for bone fracture. The authors suggest that antiresorptive therapy might be most effective in this period.

Katherine Sole

Original article Greenspan SL et al. (2005) Bone loss after initiation of androgen deprivation therapy in patients with prostate cancer. J Clin Endocrinol Metab 90: 6410-6417

\section{Optimal timing of postoperative evaluations for patients with treated acromegaly}

Acromegaly secondary to pituitary adenoma can be cured by trans-sphenoidal adenomectomy. Cure is defined by normalization of insulin-like growth factor 1 (IGF-1) levels and confirmed by growth-hormone $(\mathrm{GH})$ nadir during an oral glucose-tolerance test (OGTT GH nadir), but the biochemical criteria for and optimal timing of these evaluations after surgery remain unclear. Feelders et al. prospectively evaluated blood samples for these parameters, and found that by 1 week after surgery, OGTT GH nadir predicted cure when a cut-off value of $0.5 \mu \mathrm{g} / \mathrm{l}$ was used. By contrast, IGF-1 levels were highly variable and did not stabilize until 12 weeks after surgery. 